\title{
Another Statistical Distribution: The Exponentiated Complementary Mukherjee-Islam Distribution
}

\author{
Oyedeji Isola Osowole, Ngozi Nzelu, and Rita Nwaka
}

\begin{abstract}
This study considered a newly proposed Exponentiated Complementary Mukherjee-Islam distribution obtained by exponentiating the Complementary MukherjeeIslam distribution. Some properties of the new distribution were derived and results from the distribution indicated that the distribution is a better alternative than its baseline distribution. The new distribution is therefore a creditable addition to the existing family of exponentiated distributions.
\end{abstract}

Index Terms - Exponentiated complementary MukherjeeIslam distribution, Complementary Mukherjee-Islam distribution, Moments, Mills ratio, Reverse hazard function, Survival function.

\section{INTRODUCTION}

A random variable, $\mathrm{X}$, according to Al-Kadim and Mahdi [1] is said have an exponentiated distribution if its cumulative distribution function (cdf) is given as:

$$
G(x)=[F(x)]^{a} ; a>1
$$

This is similar to the definition of an exponentiated distribution by Lemonte, et al [2]. They noted that the probability density function (pdf) of a random variable $\mathrm{X}$ having an exponentiated distribution is given as:

$$
g(x)=a[F(x)]^{a-1} f(x) ; a>1
$$

where $f(x)$ and $F(x)$ are the baseline (parent) pdf and cdf.

Lemonte et. al. [2] observed that "generalized distributions have been widely used in statistics and many researchers have developed different methods of generalizations" from selected parent (baseline) distributions. The exponentiation technique has attracted the attention of authors because it can be used to generate generalized distributions quickly. This assertion is corroborated by Ramires et. al. [3]. They noted that it is important to study exponentiated distributions in order to provide a simpler way to derive some properties of more complex distributions. Examples of some existing exponentiated distributions in literature include: the exponentiated exponential-Poisson distribution by BarretoSouza and Cribari-Neto [4]; exponentiated Pareto

Published on January 25, 2021.

Oyedeji Isola Osowole.

(e-mail: academicprofessor2013@gmail.com)

Ngozi Nzelu.

(e-mail: ngozinzelu@ gmail.com)

Rita Nwaka.

(e-mail: renu.rita@gmail.com). distribution by Shawky et. al. [5]; exponentiated exponential-geometric distribution by Silva et. al. [6]; exponentiated generalized inverse Gaussian distribution by Lemonte and Cordeiro [7]; exponentiated Kumaraswamy distribution by Lemonte et. al. [2]; exponentiated Kumaraswamy-Dagum distribution by Huang and Oluyede [8]; exponentiated generalized extended exponential distribution by Thiago et. al. [9]; exponentiated generalized exponential Dagum distribution by Nasiru et. al. [10]; exponentiated Chen distribution by Dey et. al. [11]; exponentiated Gumbel distribution by Dey et. al. [12]; exponentiated Inverse Rayleigh distribution by Rao and Mbwambo [13]; exponentiated uniform distribution by Ramires et. al. [3]; and beta exponentiated modified weibull distribution by Shahzad et. al. [14].

In line with the observation of Ramires et. al. [3] above, this study is an attempt to propose the Exponentiated Complementary Mukherjee-Islam Distribution (ECMID) with the aim of demonstrating the flexibility of the exponentiation method and to further increase the choices available to researchers interested in exponentiated distributions.

\section{THE EXPONENTIATED COMPLEMENTARY MUKHERJEE- ISLAM DISTRIBUTION}

The baseline distribution selected for this study is the Complementary Murhkerjee-Islam distribution defined below:

$$
f(x)=b c(x c)^{b-1}, 0 \leq x \leq \frac{1}{c} ; b, c \geq 1
$$

For (3), the cumulative distribution function (c. d. f) is given as:

$$
F(x)=(x c)^{b}, 0 \leq x \leq \frac{1}{c} ; b, c \geq 1
$$

The Exponentiated Complementary Mukherjee-Islam Distribution (ECMID) using (1) and (2) is a continuous density function with cdf and pdf defined as:

$$
\begin{aligned}
& G(x)=\left[(x c)^{b}\right]^{a}, 0 \leq x \leq \frac{1}{c} ; b, c \geq 1 ; a>1 \\
& g(x)=\frac{d}{d x}[G(x)]=a b c^{a b} x^{a b-1}, 0 \leq x \leq \frac{1}{c} ; b, c \geq 1 ; a>1
\end{aligned}
$$


The plots of the cdf and pdf of the Exponentiated Complementary Mukherjee-Islam Distribution are shown below in Fig. 1 and 2.

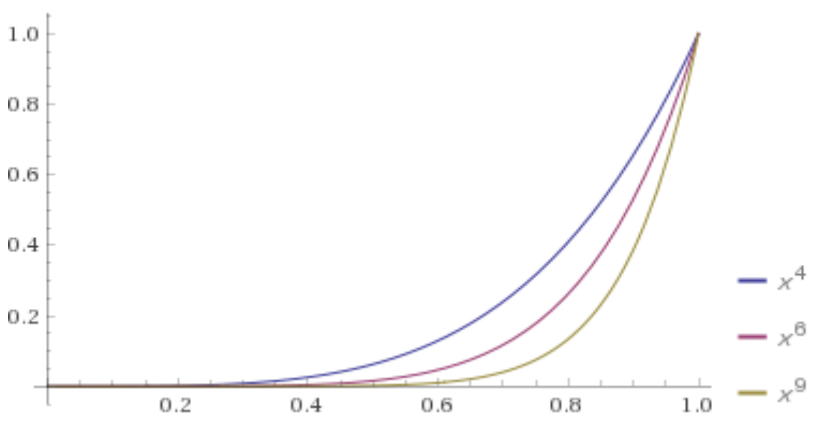

Fig. 1. The cdf plot of the ECMID at different values of the parameters for $0<\mathrm{x}<1$.

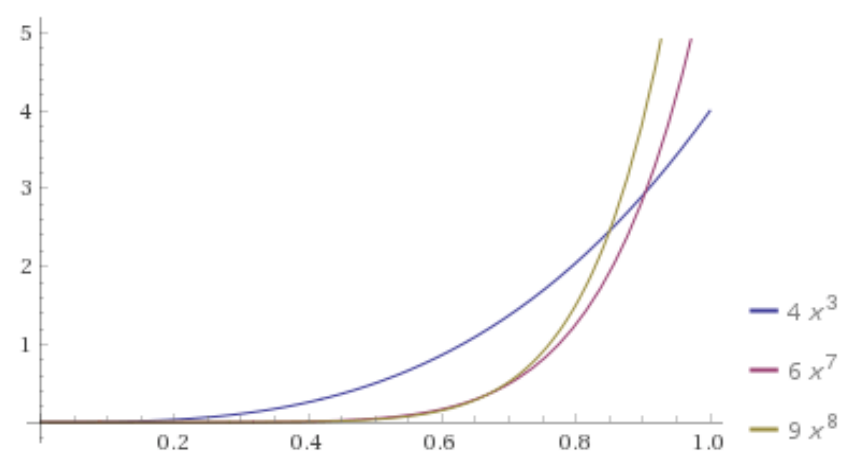

Fig. 2. The pdf plot of the ECMID at different values of the parameters for $0<\mathrm{x}<1$.

Some essential properties of the Exponentiated Complementary Mukherjee-Islam distribution shall be considered next.

\section{CHARACTERIZATION OF THE EXPONENTIATED COMPLEMENTARY MUKHERJEE-ISLAM DISTRIBUTION}

\section{A. Moments}

The $\mathrm{k}^{\text {th }}$ moment about zero for a random variable $\mathrm{X}$ from the Exponentiated Complementary Mukherjee-Islam Distribution is:

$$
\begin{aligned}
\mu_{k}^{1}=E\left(X^{K}\right) & =\int_{0}^{\frac{1}{c}} x^{k} g(x) d x \\
& =\int_{0}^{\frac{1}{c}} x^{k}\left[a b c^{a b} x^{a b-1}\right] d x \\
& =\frac{a b c^{a b}\left(\frac{1}{c}\right)^{a b+k}}{a b+k} \\
& =\frac{a b c^{a b}}{c^{a b+k}(a b+k)}, k \geq 1
\end{aligned}
$$

Specifically,

$$
\mu_{1}^{1}=\mathrm{E}(X)=\text { mean }=\frac{a b c^{a b}\left(\frac{1}{c}\right)^{a b+1}}{a b+1}
$$

The $\mathrm{k}^{\text {th }}$ moment about the mean for a random variable $\mathrm{X}$ from the Exponentiated Complementary Mukherjee-Islam Distribution is defined as:

$$
\begin{aligned}
& \mu_{k}=\mathrm{E}(X-\mu)^{k}= \\
& =\int_{0}^{\frac{1}{c}}(x-\mu)^{k} g(x) d x=\int_{0}^{\frac{1}{c}}(x-\mu)^{k}\left[a b c^{a b} x^{a b-1}\right] d x
\end{aligned}
$$

When $\mathrm{k}=2, \mu_{k}=\mu_{2}$, that is variance of $X, \sigma_{X}^{2}$. Hence,

$$
\begin{aligned}
\text { Variance }(X) & =\sigma_{X}^{2}=\mathrm{E}\left(X^{2}\right)-[\mathrm{E}(X)]^{2} \\
& =-\frac{a b\left(\frac{1}{c}\right)^{a b} c^{a b-2}\left(a^{2} b^{2}\left(\frac{1}{c}\right)^{a b} c^{a b}-a^{2} b^{2}+2 a b\left(\frac{1}{c}\right)^{a b} c^{a b}-2 a b-1\right)}{(a b+1)^{2}(a b+2)} \\
& \approx \frac{a b}{(a b+2)(a b c+c)^{2}}, \text { since }(a, b, c) \text { are positive }
\end{aligned}
$$

\section{B. Moment Generating Function}

The moment generating function (m.g.f.) of a random variable $\mathrm{X}$ from the Exponentiated Complementary Mukherjee-Islam distribution is defined as

$$
\begin{aligned}
M_{X}(t)=\mathrm{E}\left(e^{t X}\right) & =\int_{0}^{\frac{1}{c}} e^{t X} g(x) d x \\
& =\int_{0}^{\frac{1}{c}}\left[1+t X+\frac{(t X)^{2}}{2 !}+\ldots\right] g(x) d x \\
& =\int_{0}^{\frac{1}{c}} \sum_{j=0}^{\infty} \frac{t^{j}}{j !} X^{j} g(x) d x
\end{aligned}
$$

$$
\begin{aligned}
& =\sum_{j=0}^{\infty} \frac{t^{j}}{j !} \mu_{j}^{1} \\
& =\sum_{j=0}^{\infty} \frac{t^{j}}{j !}\left[\frac{a b c^{a b}\left(\frac{1}{c}\right)^{a b+j}}{a b+j}\right] ;(b, c) \geq 1 ; a>1
\end{aligned}
$$

\section{Characteristic Function}

The characteristic function (c. f.) of a random variable $\mathrm{X}$ from the Exponentiated Complementary Mukherjee-Islam Distribution is defined as:

$$
\begin{aligned}
\phi_{x}(t) & =M_{X}(i t) \\
& =\mathrm{E}\left(e^{i t X}\right) \\
& =\int_{0}^{\frac{1}{c}} e^{i t X} g(x) d x \\
& =\sum_{j=0}^{\infty} \frac{(i t)^{j}}{j !} \mu_{j}^{\prime} \\
& =\sum_{j=0}^{\infty} \frac{(i t)^{j}}{j !}\left[\frac{a b c^{a b}\left(\frac{1}{c}\right)^{a b+j}}{a b+j}\right] ;(b, c) \geq 1 ; a>1
\end{aligned}
$$

\section{Cumulant Generating Function}

The cumulant generating function (c. g. f.) of a random variable $\mathrm{X}$ from the Exponentiated Complementary Mukherjee-Islam Distribution is defined as: 


$$
\begin{aligned}
K_{X}(t) & =\operatorname{In}\left[M_{X}(t)\right] \\
& =\operatorname{In}\left[\sum_{j=o}^{\infty} \frac{t^{j}}{j !} \mu_{j}^{1}\right] \\
& =\operatorname{In}\left[\sum_{j=0}^{\infty} \frac{t^{j}}{j !}\left[\frac{a b c^{a b}\left(\frac{1}{c}\right)^{a b+j}}{a b+j}\right] ;(b, c) \geq 1 ; a>1\right.
\end{aligned}
$$

\section{E. Coefficients of Variation and Dispersion}

The coefficients of variation and dispersion for the Exponentiated Complementary Mukherjee-Islam Distribution are defined as:

$$
C V=\frac{\sigma}{E(X)} \text { and } C D=\frac{\sigma^{2}}{E(X)}
$$

Specifically,

$$
C V=\frac{(a b+1) \sqrt{\frac{a b}{(a b+2)(a b c+c)^{2}}}}{a b c^{a b}\left(\frac{1}{c}\right)^{a b+1}}
$$

and

$$
C D=\frac{\frac{(a b)(a b+1)}{(a b+2)\left(a b c+c^{2}\right)}}{\left(a b c^{a b}\left(\frac{1}{c}\right)^{a b+1}\right.}
$$

\section{F. Hazard Function}

The hazard function (h. f.) of a random variable $\mathrm{X}$ from the Exponentiated Complementary Mukherjee-Islam Distribution is defined as:

$$
\begin{aligned}
h(x) & =\frac{g(x)}{1-G(x)} \\
& =\frac{a b c^{a b} x^{a b-1}}{1-\left[(x c)^{b}\right]^{a}} ; 0 \leq x \leq \frac{1}{c} ;(b, c) \geq 1 ; a>0
\end{aligned}
$$

The plot of the hazard function for the Exponentiated Complementary Mukherjee-Islam distribution is given below in Fig. 3.

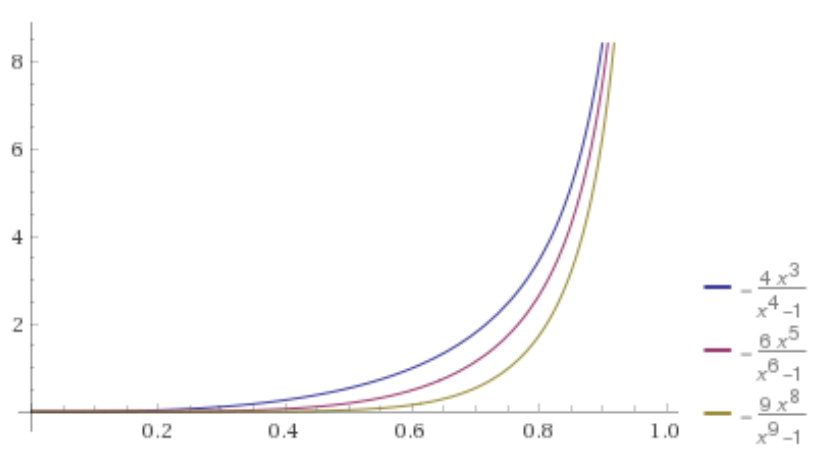

Fig. 3. The plot of the hazard function of the ECMID at different values of the parameters for $0<\mathrm{x}<1$.
The plot of the hazard function as shown above is $\mathrm{J}$ shaped at different values of the parameters and for $0<x<1$.

\section{G. Reverse Hazard Function}

The reserve hazard function (r. h. f.) of a random variable $\mathrm{X}$ from the Exponentiated Complementary Mukherjee-Islam Distribution is defined as:

$$
\begin{aligned}
h_{r}(x) & =\frac{g(x)}{G(x)} \\
& =\frac{a b c^{a b} x^{a b-1}}{\left[(x c)^{b}\right]^{a}} ; 0 \leq x \leq \frac{1}{c},(b, c) \geq 1 ; a>1
\end{aligned}
$$

The plot of the reverse hazard ratio is shown in Fig. 4 below.

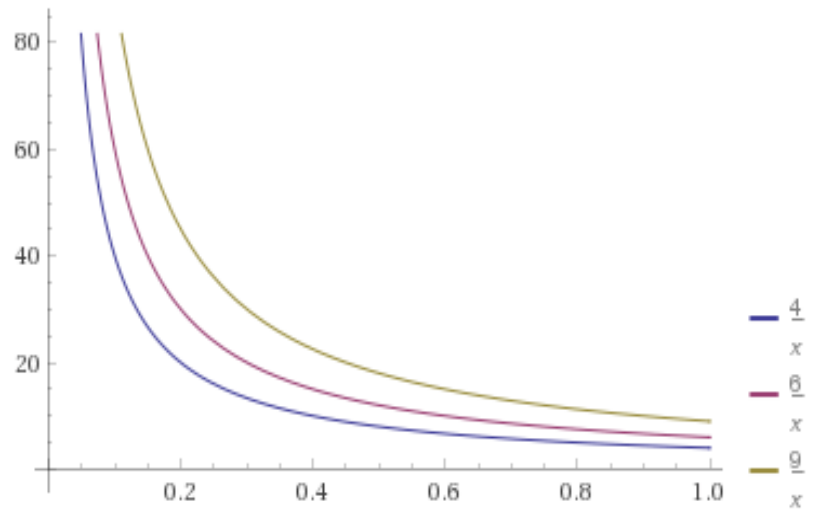

Fig. 4. The plot of the reverse hazard function of the ECMID at different values of the parameters for $0<\mathrm{x}<1$.

The plot of the reverse hazard function as shown above shows a reversed $\mathrm{J}$ shape at different values of the parameters and for $0<\mathrm{x}<1$.

\section{H. Mills Ratio}

The Mills Ratio of the Exponentiated Complementary Mukherjee-Islam Distribution is defined as:

$$
\left(\frac{1}{\text { reserve hazard function }}\right)=\left(\frac{\left[(x c)^{b}\right]^{a}}{\left(a b c^{a b} x^{a b-1}\right)}\right) ; 0 \leq x \leq \frac{1}{c},(b, c) \geq 1, a>1
$$

The plot of the Mills Ratio is given below in Fig. 5.

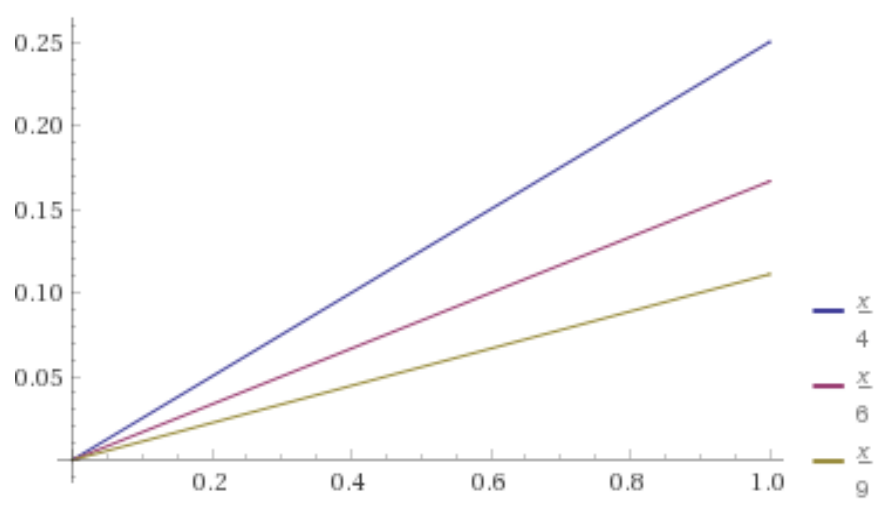

Fig. 5. The plot of the Mills Ratio of the ECMID at different values of the parameters for $0<\mathrm{x}<1$.

The plot of the Mills Ratio as shown above shows a linear function at different values of the parameters and for $0<x<1$. 


\section{Survival Function}

The survival function (s.f.) of a random variable $\mathrm{X}$ from the Exponentiated Complementary Mukherjee-Islam distribution is defined as:

$$
\begin{aligned}
S_{X}(x) & =1-G(x) \\
& =1-\left[(x c)^{b}\right]^{a} ; 0 \leq x \leq \frac{1}{c},(b, c) \geq 1 ; a>1
\end{aligned}
$$

The plot of the survival function is shown below in Fig. 6 .

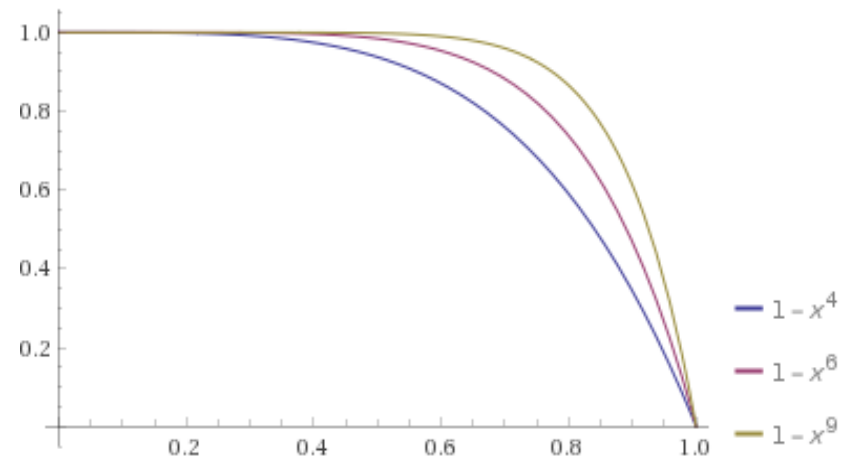

Fig. 6. The plot of the survival function of the ECMID at different values of the parameters for $0<x<1$.

\section{J. Order Statistics}

Suppose $\mathrm{X}_{(1)}, \mathrm{X}_{(2)}, \ldots, \mathrm{X}_{(\mathrm{n})}$ are the order statistics from the random sample $\mathrm{X}_{1}, \mathrm{X}_{2}, \ldots, \mathrm{X}_{\mathrm{n}}$ from the Exponentiated Complementary Mukherjee-Islam Distribution with $g(x)$ and $G(x)$ as the pdf and cdf, the pdf of the $\mathrm{r}^{\text {th }}$ order statistic where $1 \leq \mathrm{r} \leq \mathrm{n}$ is given as:

$$
\begin{aligned}
h_{(r)}(x) & =\frac{n !}{(r-1) !(n-r) !} g(x)[G(x)]^{r-1}[1-G(x)]^{n-r} \\
& =\frac{n !}{(r-1) !(n-r) !}\left[a b c^{a b} x^{a b-1}\right]\left[(x c)^{a b}\right]^{r-1}\left[1-(x c)^{a b}\right]^{n-r} ; 0 \leq x \leq \frac{1}{c},(b, c) \geq 1, a>1
\end{aligned}
$$

By setting $\mathrm{r}=\mathrm{n}$ and $\mathrm{r}=1$ in the function $\left(\mathrm{h}_{(\mathrm{r})}(\mathrm{x})\right)$ above, we have the distributions for the largest and lowest order statistics. For the largest order statistic, we have that:

$$
h_{(r=n)}(x)=\frac{n !}{(n-1) !}\left[(x c)^{a b}\right]^{n-1} a b c^{a b} x^{a b-1} ; 0<x<\frac{1}{c},(b, c) \geq 1, a>1
$$

For the lowest order statistic, we have that:

$$
h_{(r=1)}(x)=n\left[1-(x c)^{a b}\right]^{n-1} a b c^{a b} x^{a b-1} ; 0<x<\frac{1}{c},(b, c) \geq 1, a>1
$$

\section{K. Random Number Generation}

By the quantile function (i.e the inverse of the cdf), random numbers can be generated for the Exponentiated Complementary Mukherjee-Islam distribution as follows: Let $\left[\left(x_{c}\right)^{b}\right]^{a}=u$ where $\mathrm{U}$ is a random variable from the Uniform $(0,1)$ distribution. The random number generation will be done by solving the equation for $\mathrm{x}$.

$\left[(x c)^{b}\right]^{a}=u$ becomes $\operatorname{In}(x c)=\operatorname{In} u / a b$ so that $\operatorname{In} x=$
$\frac{\operatorname{In} u}{a b}-I n c$. This implies that $=e^{\frac{\operatorname{In} u}{a b}-\operatorname{In} c}$. The random number generation will be considered for $a=2, b=2$ and $\mathrm{c}=1$ for numerical illustration.

\section{RESUlT AND DisCUSSION}

TABLE I: THEORETICAL RESULTS FROM THE ECMID

\begin{tabular}{cc}
\hline Summary Statistics & Estimate \\
\hline mean & 0.80 \\
median & $0.8409(0.8)$ \\
variance & $0.0267(0.03)^{*}$ \\
standard & $0.1634(0.2)$ \\
$\begin{array}{c}\text { deviation } \\
\text { coefficient of } \\
\text { variation } \\
\text { coefficient of } \\
\text { dispersion }\end{array}$ & $0.2043(0.2)$ \\
\hline
\end{tabular}

Figures in (.) and (.) ${ }^{*}$ represent approximations to 1 and 2 decimal places.

Table I above gives the estimates of some summary statistics from the Exponentiated Complementary

\begin{tabular}{|c|c|c|}
\hline \multicolumn{3}{|c|}{ Simulated Results } \\
\hline $\begin{array}{c}\text { Sample } \\
\text { Size }\end{array}$ & $\begin{array}{l}\text { Summary } \\
\text { Statistics }\end{array}$ & Estimate \\
\hline \multirow{6}{*}{1000} & mean & $0.7941(0.8)$ \\
\hline & median & $0.8241(0.8)$ \\
\hline & variance & $0.0265(0.03)^{*}$ \\
\hline & Standard deviation & $0.1627(0.2)$ \\
\hline & coefficient of variation & $0.2049(0.3)$ \\
\hline & coefficient of dispersion & $0.0334(0.03)^{*}$ \\
\hline \multirow{6}{*}{2000} & mean & $0.7992(0.8)$ \\
\hline & median & $0.8424(0.8)$ \\
\hline & variance & $0.0266(0.03)^{*}$ \\
\hline & Standard deviation & $0.1630(0.2)$ \\
\hline & coefficient of variation & $0.2040(0.2)$ \\
\hline & coefficient of dispersion & $0.0333(0.03)^{*}$ \\
\hline \multirow{6}{*}{3000} & mean & $0.7999(0.8)$ \\
\hline & median & $0.8380(0.8)$ \\
\hline & variance & $0.0263(0.03)^{*}$ \\
\hline & Standard deviation & $0.1622(0.2)$ \\
\hline & coefficient of variation & $0.2028(0.2)$ \\
\hline & coefficient of dispersion & $0.0329(0.03)^{*}$ \\
\hline \multirow{6}{*}{5000} & mean & $0.7978(0.8)$ \\
\hline & median & $0.8388(0.8)$ \\
\hline & variance & $0.0277(0.03)^{*}$ \\
\hline & Standard deviation & $0.1666(0.2)$ \\
\hline & coefficient of variation & $0.2088(0.2)$ \\
\hline & coefficient of dispersion & $0.0347(0.03)^{*}$ \\
\hline
\end{tabular}
Mukherjee-Islam Distribution (ECMID). The approximated estimates to 1 and 2 decimal places are also shown in brackets. These theoretical estimates were obtained for $\mathrm{a}=2$, $\mathrm{b}=2$ and $\mathrm{c}=1$.

TABLE II: SIMULATION RESULTS FROM THE ECMID Figures in (.) and (.) represent approximations to 1 and 2 decimal places.

The simulation results in Table II are the simulated results obtained when $\mathrm{a}=2, \mathrm{~b}=2$ and $\mathrm{c}=1$ respectively for sample sizes 1000, 2000, 3000 and 5000. There is a concordance between the theoretical and simulated results as shown in Tables I and II for the selected summary statistics. This implies that the theoretical characterizations of the Exponentiated Complementary Mukherjee-Islam Distribution presented earlier in this study are valid for the proposed distribution. The improvement of the proposed distribution over the parent (baseline) distribution was 
further validated using the criteria of Akaike Information Criterion (AIC), Corrected Akaike Information Criterion (AICC), Bayesian Information Criterion (BIC) and Consistent Akaike Information Criterion (CAIC) as shown in Table III below. Lower estimates of these criteria generally suggest an improvement.

TABLE III: ESTIMATES OF AIC, AICC AND BICC AND CAIC FOR COMPARISON BETWEEN DISTRIBUTIONS

\begin{tabular}{|c|c|c|c|c|}
\hline & \multicolumn{4}{|c|}{ Comparison Criteria } \\
\hline & AIC & AICC & $\mathrm{BICC}$ & CAIC \\
\hline $\begin{array}{c}\text { Parent } \\
\text { Distribution: } \\
\text { (Complementary } \\
\text { Mukherjee-Islam } \\
\text { Distribution) }\end{array}$ & -202.206 & -202.182 & -193.777 & -202.182 \\
\hline $\begin{array}{c}\text { Proposed } \\
\text { Distribution: } \\
\text { (Exponentiated } \\
\text { Complementary } \\
\text { Mukherjee-Islam } \\
\text { Distribution) }\end{array}$ & -647.111 & -647.063 & -634.468 & -647.063 \\
\hline
\end{tabular}

Estimates of AIC, AICC, BIC and CAIC as shown in Table III above reveal that the proposed Exponentiated Complementary Mukherjee-Islam Distribution is a better alternative than its parent (baseline) distribution, the Complementary Mukherjee-Islam Distribution. The estimates of the four criteria of AIC, AICC, BIC and CAIC were consistently lower for the proposed distribution when compared with the estimates from the parent (baseline) distribution. This indicates a better fit based on their lower values. This is similar to the findings observed by Lemonte, et. al. [2] and Al-Kadim and Mahdi [1].

\section{CONCLUSION}

The Exponentiated Complementary Mukherjee-Islam Distribution has been derived successfully in this study as well as some of its essential properties. The method of exponentiation was employed to generate the proposed distribution from the baseline Complementary MukherjeeIslam distribution. The goodness of fit criteria of AIC, AICC, BIC and CAIC showed that the proposed distribution did well above the parent (baseline) distribution. The simulated results from the new distribution indicate that the new distribution has the potentials of a generalized distribution and could therefore be added to the existing pool of exponentiated generalized distributions.

\section{REFERENCES}

[1] Al-Kadim, K. A, and Mahdi, A. A. (2018). Exponentiated Transmuted Exponential Distribution. Journal of Babylon University Pure and Applied Science, Vol. 26(2):78-90.

[2] Lemonte, A. J., Barreto-Souza, W. and Cordeiro, G. M. (2013). The exponentiated Kumaraswamy distribution and its log-transform. Brazilian Journal of Probability and Statistics, 27(1):31-53.

[3] Ramires, T. G., Nakamura, L. R., Righetto, A. J., Pescim, R. R. and Telles, T. S. (2019). Exponentiated uniform distribution: An interesting alternative to truncated models. Semina: Ciencias Exatas e Tecnológicas, Londrina, v. 40(2): 107-114.

[4] Barreto-Souza, W. and Cribari-Neto, F. (2009). A generalization of the exponential-Poisson distribu- tion. Statistics and Probability Letters, 79: 2493-2500. MR2556316.
[5] Shawky, A. I. and Abu-Zinada, H. (2009). Exponentiated Pareto Distribution: Different Method of Estimations. International Journal of Contemporary Mathematical Sciences, 4(14):677-693.

[6] Silva, R. B., Barreto-Souza, W. and Cordeiro, G. M. (2010). A new distribution with decreasing, increasing and upside-down bathtub failure rate. Computational Statistics and Data Analysis, 54: 935-944. MR2580928.

[7] Lemonte, A.J. and Cordeiro,G.M. (2011). The exponentiated generalized inverse Gaussian distribution. Statistics and Probability Letters, 81(4): 506-517.

[8] Huang, S., Oluyede, B. O. (2014). Exponentiated KumaraswamyDagum distribution with applications to income and lifetime data. $J$. Stat. Distrib. Appl. 1 (8): 1-20.

[9] Thiago A. N. de Andrade, M. Bourguignon and G. M. Cordeiro (2016). The exponentiated generalized extended exponential distribution. Journal of Data Science 14: 393-414.

[10] Nasiru, S., Mwita, P. N., \& Ngesa, O. (2019). Exponentiated generalized exponential Dagum distribution. Journal of King Saud University-Science, 31(3), 362-371.

[11] Dey, S., Kumar, D., Ramos, P. L., \& Louzada, F. (2017a) Exponentiated Chen distribution: Properties and estimation. Communications in Statistics-Simulation and Computation, 46 (10): 8118-8139.

[12] Dey, S., Raheem, E., Mukherjee, S., \& Ng, H. K. T. (2017b). Two parameter exponentiated Gumbel distribution: properties and estimation with flood data example. Journal of Statistics and Management Systems, 20 (2): 197-233.

[13] Rao, G. S. and Mbwambo, S. (2019). Exponentiated Inverse Rayleigh Distribution and an Application to Coating Weights of Iron Sheets Data. Journal of Probability and Statistics, 2019:1-13. Article ID 7519429.

[14] Shahzad, M. N., Ullah, E., \& Hussanan, A. (2019). Beta exponentiated modified Weibull distribution: Properties and application. Symmetry, 11 (6): 1-13. 781. 